\title{
Baciloscopia positiva persistente en fase avanzada de la terapia anti-tuberculosa: No siempre indica fracaso del tratamiento
}

\author{
Adriana M. D’Alessandro H., Carlos Mogollón y Jacobus H. de Waard
}

\section{Persistent positive baciloscopy after several months of tuberculosis therapy: Not always it represents a treatment failure}

International agencies for tuberculosis (TB) control (WHO, IUATLD, KNCV) consider the presence of acid-fast bacilli (AFB) in the sputum smear after completion of TB therapy as treatment failure. We describe a case of a pulmonary TB patient who received and correctly completed 6 months of antituberculous treatment with a positive AFB sputum smear. Treatment was continued and sputum cultures were performed for resistance determination. After 9 months, despite the persistence of AFB in the sputum smear, the treatment was suspended because none of the sputum samples yielded a positive culture, indicating no viable bacilli. In addition, the patient was asymptomatic and had recovered his body weight. An exhaustive review of medical literature allowed us to conclude that a positive sputum smear after therapy has been detected in up to $5 \%$ of cases and does not always represent a treatment failure as defined by international guidelines. For scenarios where culture methods are not available we propose a scheme to evaluate these patients. This includes compliance with the treatment, severity of the disease at the moment of diagnosis, clinical symptoms after specific therapy and rate of in vitro resistance of $M$. tuberculosis in the community.

Key words: tuberculosis, baciloscopy, resistance, culture, treatment failure.

Palabras clave: tuberculosis, baciloscopia, resistencia, cultivo, fracaso de tratamiento.

\section{Introducción}

$\mathrm{E}$ n Venezuela, el tratamiento estándar de la tuberculosis (TBC) en todas sus formas consiste en dos meses de la primera fase o fase intensiva, cinco días por semana, con cuatro fármacos antituberculosos: isoniacida, rifampicina, etambutol y pirazinamida, seguido por una segunda fase, la fase de mantenimiento, con cuatro meses de isoniacida y rifampicina, tres veces por semana. El primer control bacteriológico se realiza a los tres meses de iniciado el tratamiento. Cuando el paciente resulta positivo por baciloscopia (BK) se continúa el tratamiento y se repite la BK a los cinco meses del mismo. Nuestras normas y también las normas internacionales en TBC definen como "fracaso de tratamiento" a la persistencia de una BK positiva a los 5 meses o más de tratamiento ${ }^{1-3}$. Se recomienda entonces, re-iniciar el tratamiento con un nuevo régimen de fármacos ${ }^{2,3}$ (estreptomicina, etambutol, etionamida y pirazinamida) y mantener hospitalizado al paciente; paralelamente el médico tratante debe solicitar pruebas de resistencia ${ }^{2,3}$.

Describimos el caso de un paciente masculino de 51 años de edad, de quien se sospechó resistencia al tratamiento anti-tuberculoso por permanecer bacilífero hasta por más de 9 meses después de iniciado el mismo. Discutimos la utilidad del cultivo para definir un "fracaso del tratamiento". Para situaciones donde el cultivo no está disponible, exponemos una serie de criterios para evaluar un paciente bacilífero persistente, finalizado el tratamiento, antes de clasificarlo como "fracaso terapéutico", hospitalizarlo e iniciar una terapia alternativa.

\section{Caso Clínico}

Se trata de un paciente masculino sin infección por VIH, con 51 años de edad. Presentaba fiebre alta, hemoptisis, dolor torácico, desnutrición grave con un peso de $43 \mathrm{~kg}$ (aproximadamente $8 \mathrm{kgs}$ por debajo de su peso normal), anemia (Hb 9 g/dl, Hto 31\%) y malestar general. Se le detectó una TBC pulmonar mediante una BK positiva. Efectuó tratamiento supervisado durante 6 meses, el que cumplió de manera regular y sin interrupciones, pese a lo cual persistió la BK positiva. Se le continuó terapia por 3 meses adicionales con los fármacos empleados en la fase de mantenimiento y fue
Caracas, Venezuela Instituto de Biomedicina Sección de Tuberculosis (AMD'A, JHdeW)

Hospital Vargas

Medicina Interna (CM)

Los autores reciben financiamiento del proyecto FONACIT y un aporte a la investigación efectuada por SHELL de Venezuela (LOCTI)

Recibido: 6 de septiembre 2007 Aceptado: 3 de marzo 2008

Correspondencia a: Jacobus H. de Waard jacobusdewaard@movistar.net.ve 
Figura 1. Radiografía de tórax P-A del paciente a los 6 meses de iniciado el tratamiento.

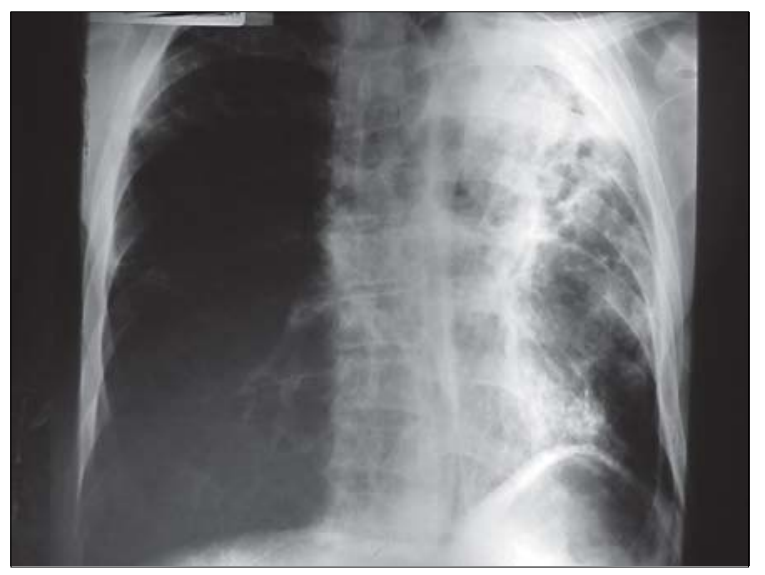

manifestaciones clínicas, la normalización de los parámetros hematológicos y la recuperación nutricional, junto a los cultivos seriados de esputo negativos y el cumplimiento regular del tratamiento por parte del paciente, eran indicativos de éxito terapéutico, aún cuando las BK persistieran positivas tras seis y nueve meses de terapia específica, (los bacilos que expulsó no eran viables).

\section{Discusión}

Definiciones internacionales en el control de la TBC, establecidas por la Organización Mundial de Salud (OMS), la International Union Against Tuberculosis and Lung Disease (IUATLD) y la Royal Netherlands Tuberculosis Association (KNCV), consideran fracaso del tratamiento anti-tuberculoso si, a los cinco meses o más de iniciado el mismo, el paciente aún tiene BK positiva ${ }^{1-3}$. En estas circunstancias, el paciente es registrado como fracaso de tratamiento e inicia un nuevo esquema terapéutico. Dichas normas enfatizan la importancia del cultivo de Koch pero no lo consideran obligatorio para la toma de decisiones. En países donde sí se realiza de rutina el cultivo, se debe utilizar este criterio para confirmar el fracaso del tratamiento, antes de iniciar el nuevo régimen. El inconveniente de esta conducta es que puede retrasar la decisión terapéutica, al menos dos meses, incrementando el riesgo de transmisión a terceros y amenazando con el deterioro de los pacientes con BK y cultivo positivos.

Por otra parte, en la literatura médica está bien documentado que un porcentaje no despreciable de pacientes con TBC pulmonar presentan BKs positivas con cultivo de esputo negativo, una vez que han finalizado el tratamiento específico. Un estudio realizado en Barcelona, España, mostró que de un total de 453 pacientes con TBC pulmonar, 10 (2,2\%) aún presentaban BKs positivas en expectoración al culminar el tratamiento. Ocho estaban asintomáticos y el resultado del cultivo fue negativo, lo que representó un éxito en el tratamiento; en los dos restantes, se identificó en el cultivo micobacterias no tuberculosas ${ }^{4}$. En Canadá se comprobó que, de 428 casos de TBC pulmonar, 22 $(5,1 \%)$ tenían BK positiva de expectoración a los seis meses de iniciada la terapia anti-tuberculosa, de los cuales seis $(27,3 \%)$ tenían cultivo positivo (genuino fracaso $)^{5}$. De estos pacientes, en dos se aisló una cepa sensible a los fármacos en uso, en otro se aisló una micobacteria atípica, un cuarto paciente tenía Mycobacterium tuberculosis resistente a isoniacida y dos tenían un aislado de $M$. tuberculosis multi-resistente. La expresión clínica en estos pacientes con cultivo positivo no está discutida en el artículo, pero sí co- 
mentan los autores que la adherencia al tratamiento fue baja en buena parte de ellos $(57 \%)^{5}$.

Ambos estudios concluyen que existen pacientes con BK positiva al finalizar el tratamiento anti tuberculoso (2,2\% y 5,1\% respectivamente), pero en la mayoría no implica un fracaso terapéutico. La BK positiva se correlaciona con la extensión de las lesiones al inicio del tratamiento; mientras más extensa es la lesión, la excreción de bacilos se prolonga en el tiempo ${ }^{4}$, y por ende, la condición de "fracaso de tratamiento" se debe definir a base de las manifestaciones clínicas, el cumplimiento del tratamiento y la radiología del paciente $e^{4,5}$. Si el paciente ha sido adherente al tratamiento, no hay una condición clínica compatible con una TBC activa y la radiología muestra mejoría, se puede concluir que el tratamiento fue exitoso. Los resultados del cultivo confirmarán esta conclusión. Si por el contrario, el cultivo aún es positivo, las pruebas de resistencia junto con la evaluación clínica, determinarán si se debe cambiar el tratamiento.

Para un mejor manejo de este tipo de pacientes que, presuntamente, también en Latinoamérica representan 2 a $5 \%$ de los casos, proponemos, en base a la literatura médica disponible, una serie de criterios, enumerados más adelante, que deben estar presentes a la hora de evaluar a un paciente, antes de pensar en fracaso del tratamiento e indicar una re-hospitalización para cambio del mismo. Aplicando estos criterios se evita que se hospitalicen pacientes ya curados y que se utilicen fármacos anti-tuberculosos de segunda línea, potencialmente tóxicos, en forma innecesaria. Por último, la relación costo-efectividad es optimizada para el programa de control de TBC.

Los criterios que proponemos, para evaluar estos pacientes conjuntamente con el especialista broncopulmonar, en espera del informe del cultivo de Koch, son:

- Condición clínica del paciente. Se determina si persisten o no, las manifestaciones clínicas en el paciente.

- Adherencia al tratamiento. Se evalúa si el paciente cumplió con la toma del tratamiento, con regularidad y sin interrupciones. (Este punto debe ser verificado con el análisis exhaustivo del correspondiente tarjetón de tratamiento).

- Radiografía de tórax. Se debe ponderar la gravedad de la enfermedad al momento de su diagnóstico. Ella se correlaciona con el tiempo que el paciente continúa siendo bacilífero; mientras más extensa es la lesión, la excreción de bacilos será más durade$\mathrm{ra}^{4,6}$. La mayoría de las veces, la baciloscopia positiva "residual” se acompaña de una mejoría radiológica.
Cuando no hay síntomas una vez finalizado el tratamiento, y el paciente cumplió cabalmente con él, se supondrá que éste fue exitoso, aún cuando continúe siendo bacilífero; probablemente esté eliminando bacilos que ya no son viables. El cultivo es crucial (estándar de oro) para decidir si hay o no un fracaso en el tratamiento. Ante la persistencia de una BK positiva, es obligatorio tomar al menos dos muestras seriadas de esputo para efectuar el cultivo de Koch. Si éste es negativo, aún cuando la BK sea positiva, no se tratará de un caso de resistencia y se catalogará el tratamiento de exitoso.

No debe omitirse en esta evaluación, la tasa de resistencia in vitro de $M$. tuberculosis en la comunidad. Cuando ésta es alta, es apropiado sospechar de una resistencia si, finalizado el tratamiento, la BK aún es positiva. En Venezuela, la tasa de resistencia primaria es baja y se ubica en $4,1 \%$, mientras que la tasa de multi-resistencia es cero ${ }^{8}$. Así, en nuevos casos de TBC un fracaso de tratamiento bien llevado, es muy poco común. Pero la tasa de resistencia secundaria (resistencia en pacientes ya tratados anteriormente por una TBC) se ubica en $16 \%$ y la multi-resistencia secundaria es de 8,3\%. La resistencia primaria suele ser común en pacientes con infección por VIH/SIDA, indígenas y en pacientes con micobacteriosis atípicas. Por esta razón, excluimos de nuestro esquema de evaluación a las personas con alto riesgo de resistencia primaria: recaídas, pacientes con infección por VIH/ SIDA, indígenas y pacientes con micobacteriosis atípicas. En estos grupos de mayor riesgo, sí es apropiado pensar en fracaso terapéutico si, al finalizar el tratamiento, la BK es aún positiva. La conducta recomendada en este caso es hospitalizar al paciente y aplicar un nuevo esquema de tratamiento, a la espera de recibir el informe del cultivo de Koch.

\section{Resumen}

Agencias internacionales para el control de la tuberculosis (TBC) (WHO, IUATLD, KNCV) consideran fracasado el tratamiento anti-tuberculoso si finalizado el mismo, persiste la baciloscopia (BK) positiva. Describimos el caso de un paciente con TBC pulmonar que, tras completar correctamente el tratamiento por 6 meses, continuaba siendo bacilífero. Después de 9 meses con terapia específica, a pesar de una BK positiva persistente, su tratamiento fue suspendido, porque estaba asintomático, con el peso corporal recuperado y ninguna muestra de esputo arrojó un cultivo positivo. Una revisión de la literatura médica permitió concluir que una BK positiva después de completar el tratamiento ocurre hasta en 5\% de los casos pero, no siempre indica un fracaso en la terapia como señalan 
definiciones internacionales. Para una mejor evaluación de este tipo de pacientes, en circunstancias en que no se dispone del cultivo, discutimos un algoritmo que toma en cuenta la gravedad de la enfermedad al ser diagnosticada, el cumplimiento del tratamiento, la condición clínica del paciente al finalizar el mismo y la tasa de resistencia in vitro de $M$. tuberculosis en la comunidad, para así definir mejor un fracaso de tratamiento.

\section{Referencias}

1.- Revised international definitions in tuberculosis control. World Health Organization. Int J Tuberc Lung Dis 2001; 5: 213-5.

2.- Ministerio de Sanidad y Asistencia Social de Venezuela. División de Tuberculosis y Enfermedades Pulmonares. Programa Nacional Integrado de Control de Tuberculosis. Normas para el Primer Nivel de Atención. Actualización 1996.

3.- Treatment for Tuberculosis. Guidelines for National Programmes. 3rd edition. http://www.who.int/tb/publications/ cds_tb_2003_313/en/ World Health Organization. WHO/TB/97.220.

4.- Vidal R, Martín-Casabona N, Juan A, Falgueras T, Miravitlles M. Incidence and significance of acid-fast bacilli in sputum smears at the end of antituberculous treatment. Chest 1996; 109(6): 1562-5.

5.- Al-Moamary M S, Black W, Bessuille E, Elwood R K, Vedal S. The significance of the persistent presence of acid-fast bacilli in sputum smears in pulmonary tuberculosis. Chest 1999; 116: 726-31.

6.- Kim T C, Blackman R S, Heatwole K M,
Kim T, Rochester D F. Acid-fast bacilli in sputum smears of patients with pulmonary tuberculosis. Prevalence and significance of negative smears pretreatment and positive smears post-treatment. Am Rev Respir Dis 1984; 129: 264-8.

7.- Gupta N. Comment on: Chest 1999; 116: 726-31. Persistent presence of acid-fast bacilli in pulmonary tuberculosis: possible implications for developing countries. Chest 2000; 118: 880-1.

8.- Anti-tuberculosis Drug Resistance in the World. Report no. 2: Prevalence and Trends WHO/CDS/TB/2000.278. 